\title{
REGULARIZATION OF SINGULAR SYSTEMS OF INTEGRAL EQUATIONS WITH KERNELS OF FINITE DOUBLE-NORM ON $L_{\infty}{ }^{1}$
}

\author{
GUILLERMO MIRANDA
}

ABstract. There are known examples of linear integral transformations $T$ of finite double-norm on $L_{\infty}$ such that neither the transformation nor any of its iterates is compact, so that Fredholm's alternative does not hold unrestrictedly for the equation $(I-\lambda T) g=f\left(\lambda\right.$ a complex number, $\left.g, f \in L_{\infty}\right)$. It is also known that the alternative holds true for $|\lambda|$ less than the Fredholm radius of $T$. Using a kernel decomposition, a quantity $\omega$ is introduced and the equivalence of an integral transformation system with components of finite double-norm on $L_{\infty}$ to a similar system that satisfies the Fredholm alternative for $|\lambda|<\omega$ is proved. In contrast to the Fredholm radius, an easy computation for $\omega$ is available.

1. Introduction. Linear integral transformations of finite doublenorm on $L_{p}$ have been considered by Zaanen [1]. We recall here the definition of such transformations.

DEFINITION. Let $\mu$ be a $\sigma$-finite measure on the set $X, T(x, y) a \mu \times \mu$ measurable function on $D \times D$, where $D$ is a $\mu$-measurable subset of $X$, and $f \in L_{p}(D, \mu), 1 \leqq p \leqq \infty$, and consider the integral transformation

$$
T f(x) \equiv \int_{D} T(x, y) f(y) d \mu_{y} .
$$

$T$ is said to be of finite double-norm on $L_{p}(D, \mu)$ whenever the number ||T $T \|$ (double-norm of $T$ ) is finite, where

$$
\||| T|| \mid \equiv\left[\int_{D}\left(\int_{D}|T(x, y)|^{q} d \mu_{\nu}\right)^{p / q} d \mu_{x}\right]^{1 / p}, \text { with } 1 / p+1 / q=1,
$$

and where the interpretation in the boundary case $p=\infty$ is evident. Note that $T$ is bounded and $\|T\| \leqq\||| T\| \|$.

In connection with corresponding integral equations, we may refer to results by Trjitzinsky [2] and Willett [3], where sufficient conditions for the convergence of successive approximations are given in

Received by the editors February 17, 1970.

AMS 1969 subject classifications. Primary 4520, 4515; Secondary 4511, 4770.

Key words and phrases. Singular systems, integral equations, Fredholm alternative, Fredholm radius, finite double-norm transformations, Neumann series, space of bounded functions.

1 Results obtained at the Courant Institute of Mathematical Sciences, New York University, under a Sloan Foundation Grant. 
the case of a single equation. These works also consider nonlinear kernels. The present paper is concerned with the validity of Fredholm's alternative for linear systems of integral equations with kernels of finite double-norm. Simple criteria for the convergence of the Neumann series are also presented in terms of the double-norms of the component kernels.

The case $p=\infty$ is especially interesting since there are examples of transformations of finite double-norm on $L_{\infty}$ such that neither the transformation nor any of its iterates is compact (see [1, p. 321]). Hence an alternative theorem is not generally available for such transformations. This stands in contrast to the case $1 \leqq p<\infty$ (at least when $\mu$ is a separable measure): if $1<p<\infty$, then $T$ is compact on $L_{p}$ into $L_{p}$, and if $p=1, T^{2}$ is compact (see [1, pp. 320 and 323]).

If $T$ is any bounded linear transformation on a Banach space $B$ and $I$ is the identity transformation, it is known that the alternative holds for the equation $(I-\lambda T) g=f$ provided $|\lambda|<\Omega, \lambda$ is a complex number, $g, f \in B$, where $\Omega$ is the Fredholm radius of $T$ (see [4, pp. 205 and 217]). Note that $\Omega \geqq 1 /\|T\|$.

When $T$ corresponds to a given system of integral transformations of finite double-norm on $L_{\infty}$, it is generally very difficult to compute $\Omega$ exactly. A certain decomposition of the kernels involved enables us to introduce an easily computable quantity $\omega$ which, while not as sharp as $\Omega$ in regard to the alternative, is sometimes better than the rather crude value $1 /\|T\|$. The system is then reduced to a regular Fredholm one, equivalent to the original singular system at least for $|\lambda|<\omega$, so that the alternative holds inside an $\omega$-circle.

The above results have been applied by the author to the solution of elliptic and parabolic boundary value problems by means of singular integral equations in the case of domains with nonsmooth boundary, i.e., corners and angular lines are admitted (see [5] and [6]).

2. Regularization of singular systems with kernels of finite doublenorm on $L_{\infty}(D, \mu)$. The following conventions will be used throughout: lower or upper case letters with a single bar on top will denote a vector in $n$-dimensional space. Upper case letters that are bold will denote $n$ by $n$ matrices. Such matrices will also be written in terms of double-indexed entries enclosed by key brackets, and the indices indicate row and column position respectively.

A product $B \bar{u}$ between a matrix $B=\left\{B_{i j}\right\}$ and a vector $\bar{u}=\left(u_{1}, \cdots, u_{n}\right)$ will denote a vector $\bar{v}=\left(v_{1}, \cdots, v_{n}\right)$ according to the usual rule: $v_{i}=\sum_{j=1}^{n} B_{i j} u_{j}$. Finally, let $\bar{B}^{(j)}=\left(B_{1 j}, \cdots, B_{n j}\right)$, $j=1, \cdots, n$, stand for the column vectors of $B$. 
Let $K_{i j}(x, y), i, j=1, \cdots, n$, be a set of kernels generating integral transformations of finite double-norm on $L_{\infty}(D, \mu)$, hence

$$
\int_{D}\left|K_{i j}(x, y)\right| d \mu_{y} \leqq\|\| K_{i j} \| \mid \quad \text { for a.e. } x \in D \text {. }
$$

$\boldsymbol{K}$ will denote the matrix of integral operators corresponding to the kernels $K_{i j}(x, y)$. Consider the following system of integral equations for the unknown functions $g_{i}(x)$, with given $f_{i}(x) \in L_{\infty}(D, \mu), i$ $=1, \cdots, n$ :

$$
(I-\lambda K) \bar{g}(x)=\bar{f}(x) \quad \text { for a.e. } x \in D,
$$

where $\lambda$ is a complex number, $\bar{f}(x)=\left(f_{1}(x), \cdots, f_{n}(x)\right)$, etc.

The kernel decomposition and regularization that follows were considered by Carleman [7], [8] in the case of a single equation. The extension of Carleman's method to systems is the content of the following

ThEOREM. Let $K(x, y)$ be a matrix of kernels satisfying (2.1) and assume that $K(x, y)=G(x, y)+H(x, y)$, where the kernels $H_{i j}(x, y)$ are essentially bounded on $D \times D$ and $(I-\lambda G)^{-1}$ exists for $|\lambda|<\omega$ (one can take $\omega^{-1}=\|M\|_{m}$ as defined in $\$ 3$ for instance). Then the singular system of integral equations (2.2) is equivalent to the regular Fredholm system $(I-\lambda Z) \bar{g}=\bar{F}$ for $|\lambda|<\omega$, where $\bar{F}=\bar{F}(x ; \lambda) \equiv(I-\lambda G)^{-1} \bar{f}(x)$ and the column vectors $\bar{Z}^{(j)}$ are defined as solutions of the auxiliary systems

$$
(I-\lambda G) \bar{Z}^{(j)}=\bar{H}^{(j)}(x, y), \quad y \text { is a parameter here, }
$$

$$
j=1, \cdots, n \text {. }
$$

Proof. Suppose $\bar{g}$ satisfies (2.2). Observing that $\boldsymbol{H}(x, y) \bar{g}(y)$ $=\sum_{j=1}^{n} g_{j}(y) \bar{H}^{(j)}(x, y)$, (2.2) becomes

$$
(I-\lambda G) \bar{g}(x)-\lambda \sum_{j=1}^{n} \int_{D} g_{j}(y) \bar{H}^{(j)}(x, y) d \mu_{y}=\bar{f}(x) .
$$

Replacing $\bar{f}(x)$ in terms of $\bar{F}(x ; \lambda)$ and $\bar{H}^{(j)}(x, y)$ as given by (2.3) $\left(\bar{F}\right.$ and $\bar{Z}^{(j)}$ are well defined for $|\lambda|<\omega$ ) one obtains

$$
\begin{aligned}
& (I-\lambda Z) \bar{g}(x)-\bar{F}(x ; \lambda)+\lambda G(\bar{F}-\bar{g})(x) \\
& \quad+\lambda^{2} \sum_{j=1}^{n} \int_{D} g_{j}(y)\left[\int_{D} G(x, z) \bar{Z}^{(j)}(z, y ; \lambda) d \mu_{z}\right] d \mu_{y}=0 .
\end{aligned}
$$

Since $\int_{D}\left|G_{i k}(x, z)\right| d \mu_{z}<\infty$ for a.e. $x \in D$ and $Z_{k j}(z, y ; \lambda)$ is essentially bounded on $D \times D$ for all $|\lambda|<\omega$, the order of integration can be interchanged in the $\lambda^{2}$-term, and (2.4) becomes 


$$
(I-\lambda G) \bar{U}(x)=0 \quad \text { for a.e. } x \in D,
$$

where

$$
\bar{U}(x) \equiv \bar{g}(x)-\lambda \int_{\boldsymbol{D}} \boldsymbol{Z}(x, y ; \lambda) \bar{g}(y) d \mu-\bar{F}(x ; \lambda) .
$$

But $|\lambda|<\omega$, so that (2.5) implies $\bar{U}(x)=0$ for a.e. $x \in D$.

Conversely, let $\bar{g}$ be a solution of $(I-\lambda Z) \bar{g}=\bar{F}$. If we form with $\bar{g}$ the expression $\bar{U}$ defined by (2.6), it is clear that $\bar{U}=0$ so that (2.5) holds. Replacing $\bar{U}$ by its definition in terms of $\bar{g},(2.5)$ becomes (2.2).

3. Neumann series solution of systems of integral equations whose component kernels generate integral transformations of finite double-norm on $L_{p}(D, \mu)$. Consider a single integral equation $(I-\lambda G) g=f$, where $G$ is an integral transformation with finite doublenorm $\||G|\|$ on $L_{p}(D, \mu), 1 \leqq p \leqq \infty$. As is well known, the Neumann series solution $g=\sum_{n=0}^{\infty} \lambda^{n} G^{n} f$ converges in $L_{p}(D, \mu)$ for $|\lambda|<1 /|||G|||$. There is no unique analogue of the above simple convergence criterion for systems of integral equations. This is due to the fact that a cartesian product of normed spaces can be endowed with a variety of norms made up from the norms in the component spaces. For instance, if $\bar{f}(x)=\left(f_{1}(x), \cdots, f_{n}(x)\right)$ with $f_{j}(x) \in L_{p}(D, \mu)$, then

$$
|\bar{f}(x)|_{m} \equiv\left(\left\|f_{1}(x)\right\| m+\cdots+\left\|f_{n}(x)\right\|^{m}\right)^{1 / m}
$$

is a norm on $L_{p} \times \cdots \times L_{p}$ for any $1 \leqq m \leqq \infty$. We shall also use ||$_{m}$ to denote the corresponding norm in numerical $n$-space when no confusion arises. Let $\bar{h}=\left(h_{1}, \cdots, h_{n}\right)$ be a vector in numerical space such that $\left\|f_{j}(x)\right\| \leqq\left|h_{j}\right|$ for all $j=1, \cdots, n$. The norm ||$_{m}$ has the obvious property $|\bar{f}(x)|_{m} \leqq|\bar{h}|_{m}$.

Consider now the system of integral equations

$$
g_{i}(x)-\lambda \sum_{j=1}^{n} \int_{D} G_{i j}(x, y) g_{j}(y) d \mu=f_{i}(x), \quad i=1, \cdots, n,
$$

where each $G_{i j}$ has finite double-norm $\left\|\left|G_{i j}\right|\right\|$ on $L_{p}(D, \mu)$. Obviously we have:

$$
\left\|\sum_{j=1}^{n} \int_{D} G_{i j}(x, y) f_{j}(y) d \mu\right\| \leqq \sum_{j=1}^{n}\left\|\mid G_{i j}\right\|\|\| f_{j} \| .
$$

Set $\boldsymbol{M} \equiv\left\{\left\|G_{i j}\right\|\right\}$ and $\bar{F} \equiv\left(\left\|f_{1}\right\|, \cdots,\left\|f_{n}\right\|\right) ;(3.2)$ implies

$$
|G \bar{f}(x)|_{m} \leqq|\boldsymbol{M} \bar{F}|_{m} \leqq\|\boldsymbol{M}\|_{m}|\bar{f}(x)|_{m},
$$


where $\|M\|_{m}$ is the norm of the linear transformation induced by $\boldsymbol{M}$ on numerical $n$-space with norm ||$_{m}$. By (3.3), the Neumann series $\bar{g}(x)=\sum_{n=0}^{\infty} \lambda^{n} G^{n} \bar{f}(x)$, solution of (3.1), converges for $|\lambda|<1 /\|M\|_{m}$. In particular,

$$
\begin{aligned}
\|M\|_{1} & =\max _{1 \leqq j \leqq n} \sum_{i=1}^{n}\left\|\left|G_{i j}\right|\right\|, \\
\|M\|_{2} & \leqq\left(\sum_{i=1}^{n} \sum_{j=1}^{n}\left\||| G_{i j}|\||^{2}\right)^{1 / 2},\right. \\
\|M\|_{\infty} & =\max _{1 \leqq i \leqq n} \sum_{j=1}^{n}\left\||| G_{i j} \mid\right\| .
\end{aligned}
$$

\section{BIBLIOGRAPHY}

1. A. C. Zaanen, Linear analysis. Measure and integral, Banach and Hilbert space, linear integral equations, Interscience, New York, North-Holland, Amsterdam and Noordhoff, Groningen, 1953. MR 15, 878.

2. W. J. Trjitzinsky, Singular non-linear integral equations, Duke Math. J. 11 (1944), 517-564. MR 6, 159.

3. D. Willett, Nonlinear vector integral equations as contraction mappings, Arch. Rational Mech. Anal. 15 (1964), 79-86. MR 28 \#2417.

4. F. Riesz and B. Sz.-Nagy, Functional analysis, Akad. Kiado, Budapest, 1953; English transl., Ungar, New York, 1955. MR 15, 132; MR 17, 175.

5. G. Miranda, Application of singular integral equation methods to static problems of non-smooth elastic bodies, Thesis, Purdue University, 1969; Notices Amer. Math. Soc. 16 (1969), 646. Abstract \#665-73.

6. - Integral equation solution of the first initial-boundary value problem for the heat equation in domains with nonsmooth boundary, Comm. Pure and Appl. Math. 23 (1970); Notices Amer. Math. Soc. 17 (1970), 171. Abstract \#672-312.

7. T. Carleman, Über das Neumann-Poincaresche problem für ein gebiet mit ecken, Thesis, Uppsala, 1916.

8. - La théorie des équations intégrales singulières et ses applications, Ann. Inst. Henri Poincare 1 (1930), 401-430.

Courant Institute of Mathematical Sciences, New York University, New YoRK, NEW YORK 10012

University of Chile, Santiago, Chile 